\title{
Linearly implicit schemes for multi-dimensional Kuramoto-Sivashinsky type equations arising in falling film flows
}

\author{
Georgios Akrivis \\ Department of Computer Science and Engineering, University of Ioannina, 45110 Ioannina, Greece \\ akrivis@cs.uoi.gr \\ Anna Kalogirou \\ Department of Mathematics, Imperial College London, London SW7 2AZ, UK \\ anna.kalogirou09@imperial.ac.uk \\ Demetrios T. Papageorgiou* \\ Department of Mathematics, Imperial College London, London SW7 2AZ, UK \\ ${ }^{*}$ Corresponding author: d.papageorgiou@imperial.ac.uk \\ and \\ Yiorgos-Sokratis Smyrlis \\ Department of Mathematics and Statistics, University of Cyprus, PO Box 20537, 1678 Nicosia, Cyprus \\ smyrlis@ucy.ac.cy
}

[Received on 23 April 2014; revised on 24 February 2015]

\begin{abstract}
This study introduces, analyses and implements space-time discretizations of two-dimensional active dissipative partial differential equations such as the Topper-Kawahara equation; this is the twodimensional extension of the dispersively modified Kuramoto-Sivashinsky equation found in falling film hydrodynamics. The spatially periodic initial value problem is considered as the size of the periodic box increases. The schemes utilized are implicit-explicit multistep (BDF) in time and spectral in space. Numerical analysis of these schemes is carried out and error estimates, in both time and space, are derived. Preliminary numerical experiments provided strong evidence of analyticity, thus yielding a practical rule-of-thumb that determines the size of the truncation in Fourier space. The accuracy of the BDF schemes (of order 1-6) is confirmed through computations. Extensive computations into the strongly chaotic regime (as the domain size increases), provided an optimal estimate of the size of the absorbing ball as a function of the size of the domain; this estimate is found to be proportional to the area of the periodic box. Numerical experiments were also carried out in the presence of dispersion. It is observed that sufficient amounts of dispersion reduce the complexity of the chaotic dynamics, and can organize solution into nonlinear travelling wave pulses of permanent form.
\end{abstract}

Keywords: Topper-Kawahara equation; linearly implicit schemes; implicit-explicit BDF schemes; spectral methods; error estimates; dynamical systems.

\section{Introduction}

In this study, we develop and implement numerical schemes to solve classes of multidimensional activedissipative partial differential equations (PDEs) in the presence of dispersion. Of particular interest are two-dimensional Kuramoto-Sivashinsky type equations arising in the hydrodynamic stability of viscous 
liquid films falling down inclined flat plates or vertical cylinders. In the case of falling film flows down a plate inclined at an angle $\theta$ to the horizontal, Topper \& Kawahara (1978) derived the following evolution equation for the shape $\eta(\xi, \zeta, r)$ of the liquid interface

$$
\eta_{T}+2 F \eta \eta_{\xi}+\frac{2 \tilde{R} F^{2}}{15} \eta_{\xi \xi}-{ }_{3}^{\tilde{R}} \Delta \eta+F \Delta \eta_{\xi}+{ }_{3}^{\tilde{R} \underline{\tilde{W}}} \Delta^{2} \eta_{=}^{0}
$$

with $\Delta=\partial^{2} / \partial \xi^{2}+\partial^{2} / \partial \zeta^{2}$ being the Laplacian in $\xi$ and $\zeta$. The dimensionless parameters appearing in (1.1) are the Froude number $F=\rho g L^{2} \sin \theta / \mu U_{0}$, the Reynolds number $\tilde{R}=\rho U_{0} L / \mu$ and the inverse Weber number $\tilde{W}=\sigma / \rho U_{0}^{2} L$. Here $g$ is the acceleration due to gravity, $L$ is a typical length (e.g., the average film thickness), $U_{0}$ is a typical flow velocity, $\rho$ is the fluid density, $\mu$ is its viscosity and $\sigma$ is the coefficient of surface tension. Clearly, $\tilde{R}$ and $\tilde{W}$ are non-negative, but $F$ can take any real value (it is negative for films wetting the underside of the inclined plate). By utilizing the transformations

$$
(\xi, \zeta)={\frac{-2 F^{2} 5 \tilde{W}}{|-5 / 2|}}^{-1 / 2}(X, Y), \quad T=\frac{75 \tilde{W}}{4 \tilde{R}\left|F^{2}-5 / 2\right|} T, \quad \eta=\frac{2^{1 / 2} \tilde{R}\left|F^{2}-5 / 2\right|^{3 / 2}}{15(5 \tilde{W})^{1 / 2} F},
$$

equation (1.1) can be rescaled into the following canonical form:

$$
H_{T}+H H_{X} \pm H_{X X}-\alpha H_{Y Y}+\delta \Delta H_{X}+\Delta^{2} H=0
$$

where the parameters $\alpha$ and $\delta$ are given by

$$
\alpha=\frac{5}{2 F^{2}-5 / 2 \mid}, \quad \delta=\frac{3^{\sqrt{ }} 5 F}{\tilde{R}\left|F^{2}-5 / 2\right| / 2 \tilde{W}} .
$$

The plus sign in the third term of (1.2) corresponds to flows above critical, i.e., $F^{2}>\frac{5}{2}$, while the minus sign to sub-critical flows $F^{2}<\frac{5}{2}$. The latter are trivial in the sense that the system is now stable to all perturbations, and it is easy to show that solutions decay exponentially to uniform trivial steady states in this case. In what follows, therefore, we consider the case $F^{2}>{ }^{5}$

We will consider the periodic initial value problem for (1.2), with the solution $H$ being periodic both in $X$ and $Y$, with periods $L_{1}$ and $L_{2}$, respectively, i.e.,

$$
H\left(X+L_{1}, Y, T\right)=H\left(X, Y+L_{2}, T\right)=H(X, Y, T) .
$$

The parameters $L_{1}$ and $L_{2}$ play an important role in the dynamics and, as they increase, they introduce more unstable modes and hence more complex dynamics.

The derivation in Topper \& Kawahara (1978) assumes angles $\theta$ away from vertical. More recently, it has been shown in Frenkel \& Indireshkumar (1999) that, when the plate is vertical, the resulting equation is identical to (1.2) but with $\alpha=0$. This case is more interesting since $\alpha>0$ introduces additional dissipation and potentially reduces the complexity of the dynamics. We also note that Indireshkumar \& Frenkel (1997) present some numerical experiments having $\alpha=0$, that indicate the presence of attractors with complex chaotic dynamics in two dimensions. 
Pinto $(1999,2001)$ studied analytically the periodic initial value problem of a special version of (1.2) that does not contain dispersion, namely,

$$
H_{T}+H H_{X}+H_{X X}+\Delta^{2} H=0 .
$$

In these papers, he proved global existence of solutions, the existence of a global attractor (i.e., a compact attracting set), and analyticity of solutions. In addition, he obtained an estimate for the radius of the global attractor in the case $L_{1}=L_{2}=2 L$; in particular, he showed that for large $L$

$$
\limsup _{T \rightarrow \infty} H(\cdot, \cdot, T)^{\prime \prime}{ }_{L^{2}}^{\mathrm{TM}} c_{1} L^{12} \ln L .
$$

Such estimates can be far from optimal, and one of the objectives of the present work is to use numerical computations to conjecture optimal bounds for both the radius of the absorbing set, as well as the band of analyticity of the solutions as $L$ increases. Our extensive numerical experiments indicate that

$$
\limsup _{T \rightarrow \infty} " H(\cdot, \cdot, T)^{\prime \prime}{ }_{L^{2}}^{\mathrm{TM}} c_{2} L \text {. }
$$

It is usual in the literature to extend the one-dimensional Kuramoto-Sivashinsky equation in the form

$$
u_{t}+\frac{1}{q}|\nabla u|^{2}+\Delta u+\Delta^{2} u=0,
$$

to be solved on periodic domains as in (1.4). This equation is mathematically interesting but, as far as we are aware, it does not arise from a concrete physical problem (equation (1.7) has been suggested as an empirical model for the process of ion-beam erosion in nanostructuring processing of materials by Frost \& Rauschenbach, 2003). Analytical studies were carried out by Sell \& Taboada (1992) and Molinet (2000), where the existence of global attractors is proved for periodic solutions in thin domains, i.e., $L_{1}$ or $L_{2}$ being small. The methods presented here can be readily extended to (1.7) and a detailed study is currently under way.

The structure of the paper is as follows. In Section 2, we carry out a numerical analysis of our schemes; these are implicit-explicit multistep schemes, that include the backward difference formulae (BDF), in time and spectral in space. In Section 3, we describe our implementation and carry out numerical experiments. Section 4 is devoted to conclusions and further discussion.

\section{Linearly implicit spectral schemes}

We find it convenient to have the same period in both variables, say $2 \pi$. To this end, we use the change of variables

$$
x:=\frac{2 \pi}{\mathrm{K}_{1}} X, \quad y:=\frac{2 \pi}{L_{2}} Y, \quad u:{ }_{2 \pi}^{L_{1}} H, \quad t:{ }_{2 \pi}={\frac{2 \pi}{L_{1}}}^{-2} T
$$

in (1.2). Subsequently, after appropriately rescaling (1.2), we write it in the form

$$
u_{t}+u u_{x}+u_{x x}-\delta_{1} \frac{v_{2}}{v_{1}} u_{y y}+\delta_{2} v_{1}^{1 / 2} u_{x x x}+\frac{v_{2}}{v_{1}} u_{x y y}-+v_{1} u_{x x x x}+2 v_{2} u_{x x y y}+\frac{\not{z}}{v_{1}} u_{y y y y}=0,
$$

with positive constants $\delta_{1}, \delta_{2}, v_{1}=\left(2 \pi / L_{1}\right)^{2}$ and $v_{2}=\left(2 \pi / L_{2}\right)^{2}$. In (2.1), $x$ is in the direction of the flow, while $y$ is the transverse coordinate. 
The time discretization of the Topper-Kawahara (TK) equation by linearly implicit multistep schemes was recently analysed in Akrivis \& Smyrlis (2011). Here, we consider fully discrete schemes, namely the combination of linearly implicit multistep schemes with spectral methods for the space discretization. We put particular emphasis on the numerical study of various physically relevant phenomena.

Remark 2.1 (Nondispersive TK equation) The third-order term

$$
u_{x x x}+\frac{v_{2}}{v_{1}} u_{x y y}
$$

in (2.1) is a dispersion term. If $u$ is a solution of the nondispersive TK equation, i.e., for vanishing $\delta_{2}$, it is easily seen that

$$
u(x, y, t):=-u(-x,-y, t)
$$

is also a solution. Thus, if the periodic initial value is odd, then $u\left(\cdot,{ }^{\prime}, t\right)$ is also odd, for all $t$ “" 0 , i.e., $u(-x,-y, t)=-u(x, y, t)$.

\subsection{Preliminaries}

We will rewrite the TK equation (2.1) in an appropriate form that will allow us to analyse its time discretization by linearly implicit schemes.

With the self-adjoint operator $A$,

$$
A^{U}:=V_{1} U_{x x x x}+2^{V_{2} U_{x x y y}}+\stackrel{\underline{Z}}{\underline{2}} U_{y y y y}+U_{x x} \quad \underline{\delta}_{1} \frac{V_{2}}{V_{1}} U_{y y}+c U,
$$

and the nonlinear operator $B$,

$$
B(U):=-\delta_{2} v^{1 / 2} U_{x x x}+\frac{v_{2}}{v_{1}} U_{x y y}^{-}-U U_{x}+c U,
$$

where $c$ is a positive constant, which will be determined later on, equation (2.1) can be written in the form

$$
u_{t}+A u=B(u)
$$

For a positive $T$, we will consider the discretization of a periodic initial value problem for (2.2), namely

$$
\begin{aligned}
& \text { \ } u_{t}+A u=B(u), \quad(x, y, t) \in \mathrm{R} \times \mathrm{R} \times(0, T), \\
& \mathbf{\lfloor}(0)=u^{0},
\end{aligned}
$$

with a given periodic initial value $u^{0}$. 
Let us first show that the operator $A$ is positive definite, when the constant $c$ is sufficiently large. We denote by $\hat{U}_{j}$ the Fourier coefficients of a $2 \pi$-periodic function $U$ of two variables,

$$
u(x, y)=\hat{U}_{j, \zeta \in Z} e^{\mathrm{i}(j x+\zeta y)} .
$$

Then, for $s \in \mathrm{R}$, we denote by $H^{s}$ the periodic Sobolev space of order $s$ in two dimensions (of period $2 \pi$ in $x$ and $y$ ) with norm ${ }^{1}$

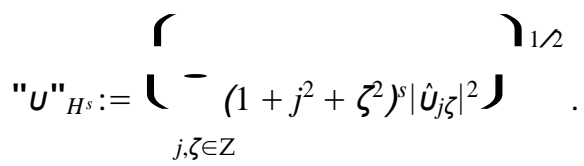

Clearly, $H^{s}$ is a Hilbert space, for every $s \in \mathrm{R}$. Let $H:=H^{0}=L^{2}$. Then the norm of $H$, which we shall be denoting by $|\cdot|$, is induced by the inner product

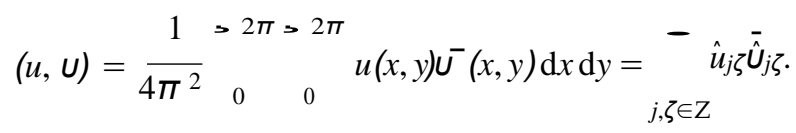

For $U \in H^{4}$, we have by periodicity

$$
(A U, U)=v_{1}\left|U_{x x}\right|^{2}+2 v_{2}\left|U_{x y}\right|^{2}+\frac{v^{2}}{v_{1}}\left|U_{y y}\right|^{2}-\left|u_{x}\right|^{2}+\delta_{1} \frac{v_{2}}{v_{1}}\left|U_{y}\right|^{2}+c|u|^{2} .
$$

Obviously,

$$
\begin{aligned}
& |u|^{2}={ }_{j, \zeta \in Z}\left|\hat{U}_{j \zeta}\right|^{2}, \quad\left|u_{x}\right|^{2}={ }_{j, \zeta \in Z} j^{2}\left|\hat{U}_{j \zeta}\right|^{2}, \quad\left|u_{y}\right|^{2}={ }_{j, \zeta \in Z}^{-} \zeta^{2}\left|\hat{U}_{j \zeta}\right|^{2},
\end{aligned}
$$

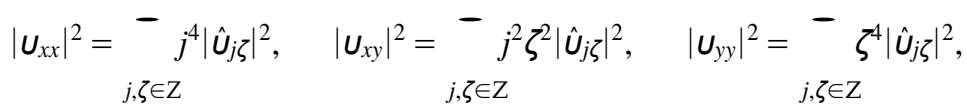

and using the obvious estimate

$$
j^{2} \text { тм } \frac{1}{4 \varepsilon}+\varepsilon j^{4},
$$

we derive the inequality

$$
\left|U_{x}\right|^{2} \text { тм } \frac{1}{4 \varepsilon}|u|^{2}+\varepsilon\left|u_{x x}\right|^{2},
$$

valid for every positive $\varepsilon$. Hence, from (2.5) we obtain

$$
\left.(A U, U) “\left(v_{1}-\varepsilon\right) \psi_{x x}\right|^{2}+2 v_{2}\left|U_{x y}\right|^{2}+\frac{2}{v_{1}}\left|U_{y y}\right|^{2}+\delta_{1} \frac{v_{2}}{v_{1}}\left|U_{y}\right|^{2}+c-\frac{1}{4 \varepsilon}{ }^{-} \mid U^{2} .
$$

\footnotetext{
${ }^{1}$ Note that, if $s$ is a non-negative integer, then ". " $H^{s}$ is equivalent to the norm defined by

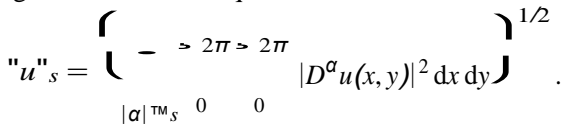


Choosing here, for instance,

$$
\varepsilon:=\frac{v_{1}}{2} \text { and } c:=1+\frac{1}{2 \downarrow},
$$

we easily see that $A$ is positive definite,

$$
(A u, u) \text { “ } \sigma " u^{\prime \prime}{ }_{2}^{2} \text { for all } u \in H^{4}
$$

with a positive constant $\sigma$.

Let " . " denote the norm of the space $V:=D\left(A^{1 / 2}\right)=H^{2}$, defined by " $u ":=\left|A^{1 / 2} U\right|$. We identify $H$ with its dual, and denote by $V^{\mathrm{r}}:=H^{-2}$ the dual of $V$, again by $\left({ }^{\prime},{ }^{\prime}\right)$ the duality pairing between $V^{\mathrm{r}}$ and $V$, and by ". " $\theta$ the dual norm on $V^{\mathrm{r}}, "{ }^{\prime}{ }_{\theta}:=\left|A^{-1 / 2} U\right|$. Obviously, $" U "=(A U, U)^{1 / 2}$ and "U" $\theta=\left(U, A^{-1} U\right)^{1 / 2}$.

Then, since $A: V \rightarrow V^{\mathrm{r}}$, from (2.7) we obtain

$$
(A u, U) \text { “ } \sigma " u^{\prime \prime}{ }_{2}^{2} \text { for all } u \in V=H^{2} .
$$

Next, we will show that the operator $B$ satisfies an appropriate local Lipschitz condition in the tube $T_{u}$ around the solution $u$ defined in terms of the $L^{\infty}$-norm, i.e.,

$$
T_{u}:=' U \in V: \min _{t} " U-u(t)^{\prime \prime}{ }_{L^{\infty}} \mathrm{TM}^{\prime \prime} .
$$

For convenience, we split $B$ into two parts, $B=B_{1}+B_{2}$, with

$$
B_{1}(U):=-U U_{x}+c U \text { and } B_{2}(U):=-\delta_{2} v_{1}^{1 / 2} U_{x x x}+\frac{v_{2}}{v_{1}} U_{x y y} \text {. }
$$

Now, for $U, U^{\sim}, w \in V=H^{2}$, we have

$$
\begin{aligned}
& \left(B_{1}(U)-B_{1}(\tilde{U}), w\right)=\frac{1}{2}\left(U^{2}-U^{2}, w_{x}\right)+c(U-\tilde{U}, w) \\
& \text { тм 专"U }+\tilde{U^{\prime \prime}} L^{\infty}|U-\tilde{U}|\left|w_{x}\right|+c|U-\tilde{U}||w| \\
& \text { тм } \frac{1}{4} " U+\tilde{U^{\prime \prime}}{ }_{L^{\infty}}{ }^{2}+c{ }^{2 \cdot 1 / 2}|U-\tilde{U}| " w^{\prime \prime}{ }_{1} \text {, }
\end{aligned}
$$



$$
" B_{1}(U)-B_{1}(\tilde{U}){ }_{\theta}{ }^{\mathrm{TM}} \mu_{1}|U-\tilde{U}| \quad \text { for all } U, \tilde{U} \in T_{u},
$$

with

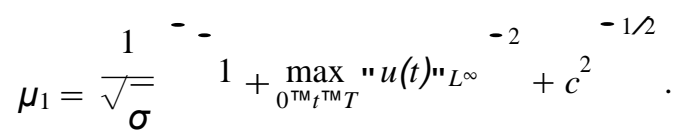

Furthermore, it is easily seenthat

$$
\left(B_{2}(U), w\right)^{\text {тм }}\left|\delta_{2}\right| v_{1}^{1 / 2} \max \overline{1},{\frac{v_{2}}{v_{1}}}^{-}{ }^{\sqrt{2}}\left|u_{x}\right| " w{ }_{2},
$$


whence, in view of(2.8),

$$
\left(B_{2}(U), w\right)^{\text {тм }} \frac{\sqrt{ } Z}{\sqrt{ }} \frac{1 / 2}{\sigma}\left|\delta_{2}\right| v_{1} \max 1, \frac{v_{2}^{-}}{v^{1}}\left|u_{x}\right| \text { "w", }
$$

i.e.,



using here (2.6) and the linearity of $B_{2}$, we obtain

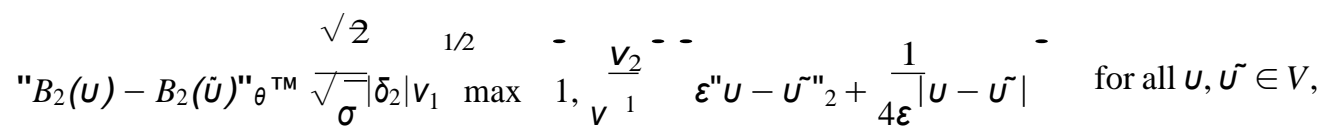

for any positive $\varepsilon$.

We infer from (2.9) and (2.10) that $B$ satisfies the local Lipschitz condition used in Akrivis et al. (1999) for any positive $\lambda$. Hence, all implicit-explicit multistep methods considered in Akrivis et al. (1999), and, indeed, the wider class of methods considered in Akrivis \& Crouzeix (2004) are suitable for the discretization of the periodic initial value problem (2.3) for the TK equation.

Remark 2.2 It follows immediately from (2.9) that it is possible for the TK equation as well to have a local Lipschitz condition with $\lambda=0$. This can be done by considering $B_{2}$ as a dispersive operator, $D:=B_{2}$, and, consequently, letting $B:=B_{1}$; see Akrivis \& Smyrlis (2011), Remark 4.2. The advantage of this splitting is that we can get by with less stringent conditions on the starting approximations; see Akrivis \& Crouzeix (2004), Remark 7.2, and Akrivis \& Smyrlis (2011), Remarks 2.2 and 3.2. More precisely, the conditions (2.12) and (2.18) on the starting approximations, respectively, can be replaced by the corresponding ones without the second term on their left-hand sides. The drawback of this splitting, however, is that we have to confine ourselves to implicit-explicit multistep schemes of first or second order; see Akrivis \& Smyrlis (2011).

\subsection{Time discretization}

We discuss here the discretization in time of the TK equation by implicit-explicit $(\alpha, \beta, \gamma)$-schemes.

Let $(\alpha, \beta)$ be a strongly $A(0)$-stable $q$-step scheme and $(\alpha, \gamma)$ an explicit $q$-step scheme, both of order $p$, characterized by the polynomials $\alpha, \beta$ and $\gamma$,

$$
\alpha(\zeta)={ }_{i=0}^{q} \alpha_{i} \zeta^{i}, \quad \beta(\zeta)=\stackrel{q}{q}_{i=0} \beta_{i} \zeta^{i}, \quad \gamma(\zeta)=\stackrel{q-1}{-} \gamma_{i} \zeta^{i}
$$

We then combine the schemes $(\alpha, \beta)$ and $(\alpha, \gamma)$, and construct an implicit-explicit $(\alpha, \beta, \gamma)$-scheme for the discretization of the TK equation (2.1) written in the form (2.2). Let $N \in \mathrm{N}, k:=T / N$ be the time step, $t^{n}:=n k, n=0, \ldots, N$, and $u$ the solution of (2.3). The linear part of the equation (2.2) is discretized by the implicit scheme $(\alpha, \beta)$ and the nonlinear part by the explicit scheme $(\alpha, \gamma)$, i.e., we 
define approximations $U^{n}$ to the nodal values $u^{n}:=u\left({ }^{\prime}, t^{n}\right)$ by the $(\alpha, \beta, \gamma)$-scheme

$$
\underset{i=0}{\underline{q}}\left(\alpha_{i} I+k \beta_{i} A\right) U^{n+i}=k \stackrel{q-1}{\stackrel{q}{i=0}} \gamma_{i} B\left(U^{n+i}\right),
$$

for $n=0, \ldots, N-q$, for given starting approximations $U^{0}, \ldots, U^{q-1}$.

In particular, we are interested in the implicit-explicit BDF schemes: For $q \in\{1,2,3,4,5,6\}$, let the polynomials $\alpha, \beta$ and $\gamma$ be given by

$$
\alpha(\zeta):={ }_{j=1}^{q}{ }^{-\frac{1}{}} \zeta^{q-j}(\zeta-1)^{j}, \beta(\zeta):=\zeta^{q} \text { and } \gamma(\zeta):=\zeta^{q}-(\zeta-1)^{q} \text {. }
$$

The corresponding $(\alpha, \beta)$-scheme is the $q$-step BDF scheme; its order is $p=q$. These schemes are strongly $A(0)$-stable. For a given $\alpha$, the scheme $(\alpha, \gamma)$ is the unique explicit $q$-step scheme of order $p=q$; the order of all other explicit $q$-step schemes $(\alpha, \tilde{V})$ is at most $q-1$. In this particular case, the general scheme (2.11) reduces to

$$
\stackrel{q}{q}_{i=0} \alpha_{i} U^{n+i}+k A U^{n+q}=k{ }_{i=0}^{q-1} \gamma_{i} B\left(U^{n+i}\right), \quad n=0, \ldots, N-q .
$$

According to Akrivis \& Crouzeix (2004), Theorem 4.1, we have the following error estimate:

Proposition 2.3 (Error estimate) Let the solution $u$ of (2.3) be sufficiently smooth. Assume we are given starting approximations $U^{0}, U^{1}, \ldots, U^{q-1} \in V \cap T_{u}$ to $u^{0}, \ldots, u^{q-1}$ such that

$$
\max _{0^{\mathrm{TM} j^{\mathrm{TM}} q-1}}\left(" u\left(\cdot, t^{j}\right)-U^{j "} L^{2}+k^{1 / 2 "} u\left(\cdot, t^{j}\right)-U^{j "} H^{2}\right)^{\mathrm{TM}} C k^{p} .
$$

Let $U^{n} \in V, n=q, \ldots, N$, be recursively defined by (2.11). Then, there exists a constant $C$, independent of $k$, such that, for $k$ sufficiently small, i.e., for $N$ sufficiently large,

$$
\max _{0^{\mathrm{TM}} n^{\mathrm{TM} N}} " u\left(\cdot, t^{n}\right)-U^{n "} L^{2}{ }^{\mathrm{TM}} C k^{p} .
$$

\subsection{Discretization in space and fully discrete schemes}

Let $M \in \mathrm{N}$ and $S_{M}:=\operatorname{span}\left\{\phi_{j \zeta}:-M+1^{\mathrm{TM}} j, \zeta^{\mathrm{TM}} M\right\}$, with $\phi_{j \zeta}(x, y):=e^{\mathrm{i}(j x+\zeta y)}$.

Let $P_{M}: V^{\mathrm{r}} \rightarrow S_{M}$ denote the orthogonal $L^{2}$-projection operator onto $S_{M}$, i.e., $\left(U-P_{M} U, X\right)=0$, for all $X \in S_{M}$. Obviously, $P_{M} U$ corresponds to the partial sum

$$
P_{M} U=\underset{j, \zeta=-M+1}{M} \hat{U}_{j, \zeta} \phi_{j, \zeta}
$$

of the Fourier series (2.4) of $U$. Since differentiation commutes with $P_{M}$, we have $P_{M} A=A P_{M}$. Furthermore, we define the discrete nonlinear operator $B_{M}: H^{2} \rightarrow S_{M}$ by $B_{M}:=P_{M} B$. 
In the semidiscrete problem corresponding to the periodic initial value problem (2.3), we seek a function $u_{M}$ such that $u_{M}(\cdot, t) \in S_{M}$, satisfying

$$
\begin{aligned}
& -\partial_{t} u_{M}(\cdot, t)+A u_{M}(\cdot, t)=B_{M}\left(u_{M}(\cdot, t)\right), \quad 0<t<T, \\
& u_{M}(\cdot, 0)=u_{M}^{0},
\end{aligned}
$$

with $u_{M}^{0} \in S_{M}$ a given approximation to $u^{0}$.

To construct implementable, fully discrete schemes, we discretize the initial value problem (2.13) for a system of o.d.e.'s in time by the implicit-explicit $(\alpha, \beta, \gamma)$-scheme, i.e., we recursively define a sequence of approximations $U^{\zeta} \in S_{M}$ to $u\left(\cdot, t^{\zeta}\right)$ by

$$
\stackrel{q}{-}\left(\alpha_{i} I+k \beta_{i} A\right) U^{n+i}=k \stackrel{q-1}{{ }_{i=0}} \gamma_{i} B_{M}\left(U^{n+i}\right),
$$

$n=0, \ldots, N-q$, for given starting approximations $U^{0}, \ldots, U^{q-1} \in S_{M}$.

\subsection{Error estimates}

The projection $P_{M}: V^{\mathrm{r}} \rightarrow S_{M}$ has the following approximation property: For $m \in \mathrm{N}$, there exists a constant $c$, independent of $U$ and $M$, such that, for $U \in H^{m}$ and $\zeta=0, \ldots, m$,

$$
\text { "U }-P_{M} U^{\prime \prime}{ }^{\mathrm{TM}} c M^{\zeta-m "} U^{\prime \prime}{ }_{H}
$$

see, e.g., Canuto et al. (1988),(9.7.4).

Let $W(\cdot, t) \in S_{M}$ denote the $L^{2}-$ projection of $u(\cdot, t)$ in $S_{M}$,

$$
W(\cdot, t)=P_{M} u(\cdot, t), \quad t \in[0, T] .
$$

Let $E_{M}(t) \in S_{M}$ denote the consistency error of the semidiscrete equation (2.13) for $W$,

$$
E_{M}(t):=W_{t}(\cdot, t)+A W(\cdot, t)-B_{M}(W(\cdot, t)), \quad t \in[0, T] .
$$

Now, using the linearity of $B_{2}$ and the fact that $P_{M}$ commutes with differentiation, we obtain

$$
E_{M}(t)=P_{M}\left[u_{t}(\cdot, t)+A u(\cdot, t)-B_{2}(u(\cdot, t))-B_{1}(W(\cdot, t))\right],
$$

and thus, in view of (2.2),

$$
E_{M}(t)=P_{M}\left[B_{1}(u(\cdot, t))-B_{1}(W(\cdot, t))\right]
$$

Now, as a consequence of (2.15), $W(\cdot, t) \in T_{u}$, for $t \in[0, T]$, and thus in view of (2.9) and (2.15), for $u(\cdot, t) \in H^{m}, t \in[0, T]$, we easily obtain the following optimal order estimate for the consistency error $E_{M}$

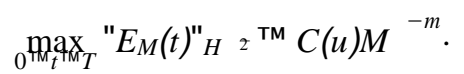

We can now derive an optimal order error estimate. 
Theorem 2.4 (Error estimate) Assume that $U^{0}, U^{1}, \ldots, U^{q-1} \in S_{M}$ are starting approximations to $u\left(\cdot, t^{0}\right), \ldots, u\left(\cdot, t^{q-1}\right)$ such that

$$
\max _{0^{\mathrm{TM} j^{\mathrm{TM}} q-1}}\left(" u\left(\cdot, t^{j}\right)-U^{j "} L^{2}+k^{1 / 2 "} u\left(\cdot, t^{j}\right)-U^{j "} H^{2}\right)^{\mathrm{TM}} c\left(k^{p}+M^{-m}\right) .
$$

Let $U^{n} \in S_{M}, n=q, \ldots, N$, be recursively defined by (2.14). Then, if the solution $u$ of (2.3) is sufficiently smooth, there exists a constant $C$, independent of $k$ and $M$, such that, for $k$ sufficiently small and $M^{4 m} k$ sufficiently large,

$$
\max _{0^{\mathrm{TM}} n^{\mathrm{TM} N}} " u\left(\cdot, t^{n}\right)-U^{n " L^{2}}{ }^{\mathrm{TM}} C\left(k^{p}+M^{-m}\right) .
$$

Proof. First of all, in view of the approximation property (2.15), we have

$$
\max _{0^{\mathrm{TM}} n^{\mathrm{TM} N}} " u\left(\cdot, t^{n}\right)-W\left(\cdot, t^{n}\right)^{\mathrm{N}} L^{2}{ }^{\mathrm{TM}} c M^{-m}
$$

and, also, for $M$ sufficiently large,

$$
\max _{0^{\mathrm{TM}} n^{\mathrm{TM} N}} " u\left(\cdot, t^{n}\right)-W\left(\cdot, t^{n}\right)^{{ }^{\prime}} L^{\mathrm{TM}} \frac{1_{4}}{4}
$$

We next let starting values $\tilde{W}^{j}:=W\left(\cdot, t^{j}\right), j=0, \ldots, q-1$, and define $\tilde{W}^{n} \in S_{M}, n=q, \ldots, N$, by applying the implicit-explicit time stepping scheme $(\alpha, \beta, \gamma)$ to equation (2.16), i.e., by



Then, according to Akrivis \& Crouzeix (2004), Theorem 4.1, see in particular the stability estimate (4.6) there, under the assumption

$$
\max _{0^{\mathrm{TM}} t^{\mathrm{TM} T}} " \partial_{t}^{j} u(\cdot, t) " H^{2}{ }^{\mathrm{TM}} C, \quad j=1, \ldots, p+1,
$$

for a constant $C$, we have

$$
\begin{aligned}
& " W\left(\cdot, t^{n}\right)-\tilde{W}_{2}^{n "}{ }_{L^{2}}+k \bar{n} " W\left(\cdot,{ }_{n}\right)-\tilde{W} \underset{n 2^{2}}{ }{ }^{\mathrm{TM}} C k_{2 p}, \\
& \zeta=0
\end{aligned}
$$

$n=q, \ldots, N$. Therefore, in particular, we infer that

$$
\max _{0^{\mathrm{TM}} n^{\mathrm{TM}} N} " W\left(\cdot, t^{n}\right)-\tilde{W}^{n "} L^{2}{ }^{\mathrm{TM}} C k^{p}
$$

and, for $k$ sufficiently small,

$$
\max _{0^{\mathrm{TM}} n^{\mathrm{TM} N} N} " W\left(\cdot, t^{n}\right)-\tilde{W}^{n "} L^{\infty}{ }^{\mathrm{TM}} \frac{1}{4}
$$

In view of (2.20) and (2.23), it remains to estimate $\vartheta^{n}:=\tilde{W}^{n}-U^{n}$. Subtracting (2.14) from (2.22), we obtain

$$
\underset{i=0}{\underline{q}}\left(\alpha_{i} I+k \beta_{i} A\right) \vartheta^{n+i}=k \stackrel{q-1}{i=0}_{i}\left[B_{M}\left(\tilde{W}^{n+i}\right)-B_{M}\left(U^{n+i}\right)\right]+k \stackrel{-}{{ }^{q-1}} \gamma_{i=0} E_{M}\left(t^{n+i}\right) .
$$


Now, using the stability estimate Akrivis \& Crouzeix (2004), (5.16), we obtain

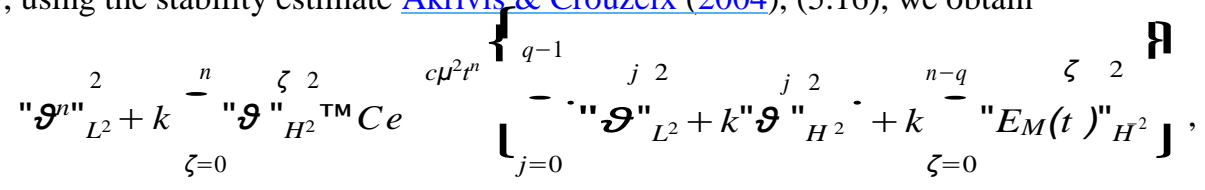

provided that $U^{0}, \ldots, U^{n-1} \in T_{u}$, with an appropriate constant $\mu$; see (2.9) and (2.10). According to (2.18) and (2.17), there exists a constant $C_{\theta}$ such that



We will use induction to prove (2.25).

We start with a preparatory statement. Assume, for the time being, that

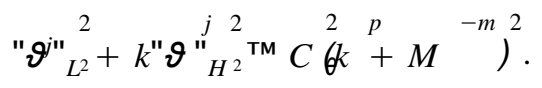

Then, using the well-known interpolation inequality

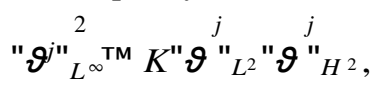

see, e.g. (Adams \& Fournier, 2003, p. 140, relation (12)), we obtain

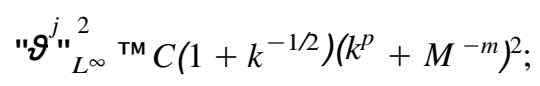

therefore, for $k$ sufficiently small and $M^{4 m} k$ sufficiently large, we have

$$
\text { " }{ }^{j "}{ }_{L^{\infty}} \mathrm{TM} 1_{\overline{2}}
$$

From (2.21), (2.24) and (2.28), we obtain

$$
" u\left(\cdot, t^{j}\right)-U^{j "}{ }_{L^{\infty}}{ }^{\mathrm{TM}} 1
$$

and infer that $U^{j} \in T_{u}$.

According to (2.18), the estimate (2.27) is valid for $j=0, \ldots, q-1$, and hence $U^{0}, \ldots, U^{q-1} \in T_{u}$. Consequently, the estimate (2.25) is valid for $n=0, \ldots, q$. Assuming that it holds for $0, \ldots, n-1$ with $q<n^{\mathrm{TM}} N$, and using (2.26), we see that (2.27) is valid for $j=0, \ldots, n-1$, and infer, as before, that $U^{0}, \ldots, U^{n-1} \in T_{u}$. Thus, (2.25) holds for $n$ as well and the induction proof is complete.

From (2.25) and (2.26), we easily conclude, for $k$ sufficiently small and $M^{4 m} k$ sufficiently large,

$$
\max _{0^{\mathrm{TM}} n^{\mathrm{TM} N}} " \tilde{W}^{n}-U^{n " L^{2} \mathrm{TM}} C\left(k^{p}+M^{-m}\right) \text {. }
$$

From (2.20), (2.23) and (2.29), the desired estimate (2.19) follows and the proof is complete.

Remark 2.5 Here, we briefly discuss two issues, the computation of starting approximations satisfying (2.18) as well as the extension of our results to more general time-stepping schemes. The computation of appropriate starting approximations in the general case of abstract parabolic equations when the discretization in space is based on the finite element method, is discussed in detail in section 6 of Akrivis \& Crouzeix (2004); the ideas presented there can be adapted to the present case of the TK 
equation with space discretization based on the spectral method, and lead to starting approximations $U^{0}, \ldots, U^{q-1} \in S_{M}$ satisfying (2.18).

To summarize, in this section we established error estimates for fully discrete schemes for the periodic initial value problem (2.3) for the TK equation. These schemes result by combining implicitexplicit multistep schemes for the time discretization with spectral methods for the discretization in space - see Theorem 2.4. Analogous results for fully discrete methods for (2.3), that use time-stepping under the wider class of linearly implicit schemes considered in Akrivis \& Crouzeix (2004), can also be easily derived. This is not pursued in any more detail due to space constraints.

\section{Numerical experiments}

\subsection{Implementation}

We use the implicit-explicit BDF schemes (2.14) of order $q=1, \ldots, 6$, to compute solutions of equation (2.2) analysed in Section 2; a list of these six schemes may be found in the appendix of Akrivis et al. (2011). Alternative schemes are available-for a comparative review as well as a new scheme for stiff PDEs, the reader is referred to Kassam \& Trefethen (2005).

We consider doubly periodic initial conditions, hence the solution takes the form

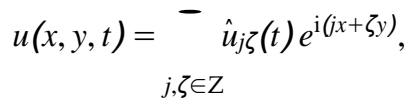

with $\hat{u}_{j \zeta}(t)$ to be computed. Note that the mean

$$
\bar{u}=\underset{(2 \pi)^{2}}{1}{ }_{0}{ }_{0}^{2 \pi>2 \pi} u(x, y, t) \mathrm{d} x \mathrm{~d} y
$$

of solutions to (2.2) is constant, and hence without loss of generality we take $\bar{u}=0$. (Note that if $\bar{u} \neq 0$, then $u(x-\bar{u} t, y, t)-\bar{u}$ is also a solution and has zero mean.) Hence, $\hat{u}_{00}(t)$ in (3.1) is zero.

Substitution of (3.1) into (2.2) provides the following infinite-dimensional dynamical system for the Fourier coefficients $\hat{u}_{j}$ :

where

$$
\stackrel{\underline{\mathrm{d}} u_{i \zeta}}{\mathrm{d} t}+\alpha_{j \zeta} \hat{u}_{j \zeta}=\beta_{j \zeta} \hat{u}_{j \zeta}-\widehat{\left(u u_{x}\right)_{j \zeta}},
$$

$$
\begin{aligned}
& \alpha_{j_{\zeta}}=v_{1} j^{4}+2 v_{2} j^{2} \zeta^{2}+\stackrel{2}{\stackrel{2}{2} \zeta} v_{1}-j^{2}+\delta_{1} \frac{v_{2}}{v_{1}} \zeta^{2}+c, \\
& \beta_{j \zeta}=\mathrm{i} \delta_{2} v_{1}^{1 / 2}-j^{3}+j \zeta_{1}^{-}+c .
\end{aligned}
$$

In the computations that follow $v_{1}=v_{2}$ (this corresponding to square boxes in the unscaled domain), we truncate system (3.2) to a finite number of modes $N$ in each spatial dimension. For efficiency, the nonlinear term on the right-hand side of (3.2) is calculated using fast Fourier transforms, and care was taken to remove any aliasing errors - we employed the classical two-thirds rule extended to twodimensional arrays, Boyd (2001). Preliminary numerical experiments showed an exponential decay of the Fourier.coefficients for $|j|+|\zeta|$ sufficiently large- see Section 3.2 for details; this in turn guided us in the efficient choice of $N$, namery $N \sim v_{1}=v_{2}$. We note that even in the presence of dispersion, 


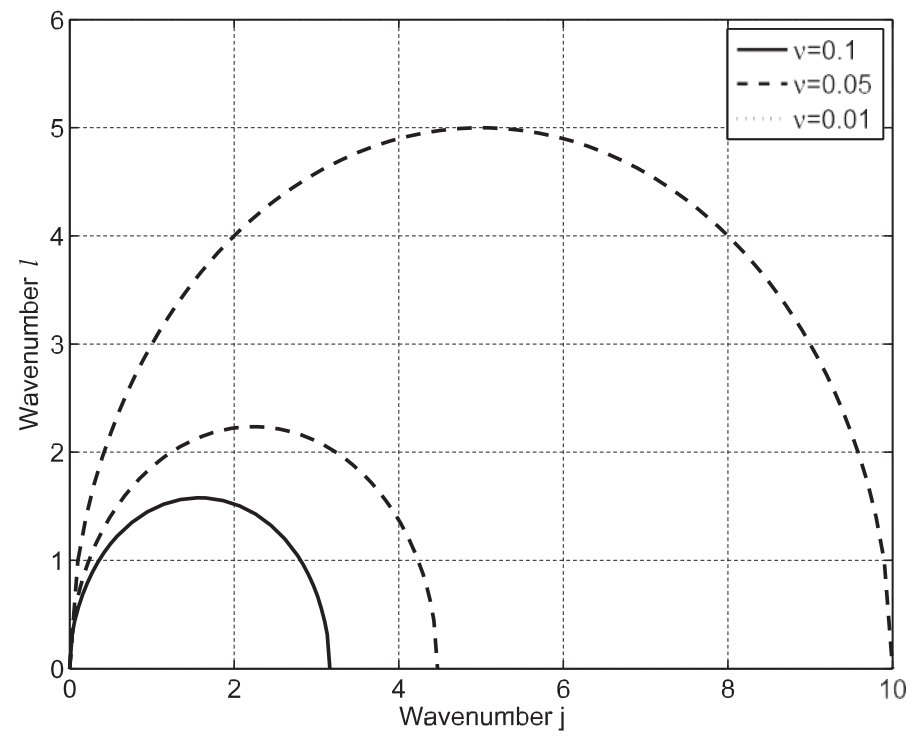

Fig. 1. Neutral stability curves for the eigenvalues $\lambda$ given by (3.3) for different values of $v$ indicated. As $v$ decreases, so does the size of the unstable region.

$\delta_{2} \neq 0$, all our schemes $(q=1, \ldots, 6)$ are stable since the dispersive terms are of lower order than the fourth order self-adjoint operator $A$.

Before proceeding to numerical experiments, we consider the linear properties of the equation. Linearization of (3.2) and looking for solutions of the form $\hat{u}_{j l} \sim e^{\lambda t}$ yields the eigenvalues (selecting $v_{1}$ $=v_{2}=v$ and $\left.\delta_{1}=\delta_{2}=0\right)$

$$
\lambda=j^{2}-v\left(j^{4}+2 j^{2} \zeta^{2}+\zeta^{4}\right)
$$

Even though $\lambda$ is defined for integer values $j, \zeta$, it is useful to consider its values as $j, \zeta$ take on any positive value in order to obtain instability regions, and it is understood that only integer pairs $(j, \zeta)$ within such regions are admissible unstable modes. Inspection of (3.3) shows that when $\zeta=0$ there isa band of unstable modes $0<j<1{ }^{2} v$, and this is the familiar result for the one-dimensional KuramotoSivashinsky equation. Depending on the value of $v$, unstable modes exist in the $y$-direction also, and we illustrate this in Fig. 1 that plots the neutral curves $\lambda=0$ for $v=0.1,0.05$ and 0.01 (with solid, long-dashed and dashed curves, respectively).

The flow is unstable below the curves and stable above them, consistent with the underlying long wave instability. Using elementary methods, we can find the maximum point of the neutral curves to be $(1 / 2 v, 1 / 2 v)$. This establishes that there are order $v^{-1 / 2}$ unstable modes in either direction and, in fact, an upper bound of the total number of unstable modes is given by the area $A$ under the neutral curves, i.e., the area of

$$
\left\{(x, y) \in \mathrm{R}^{2}: x, y \text { “ } 0 \quad \text { and } \quad x^{4}+2 x^{2} y^{2}+y^{4}-x^{2} / V^{\text {TM }} 0\right\}
$$

In fact, this area can be found using elementary methods and the result is $A=\pi / 8 \sqrt{ } \bar{v}$. We can conclude, therefore, that as $v$ decreases we expect to find complex dynamics in both directions, and in the remainder of this study we consider these cases. 


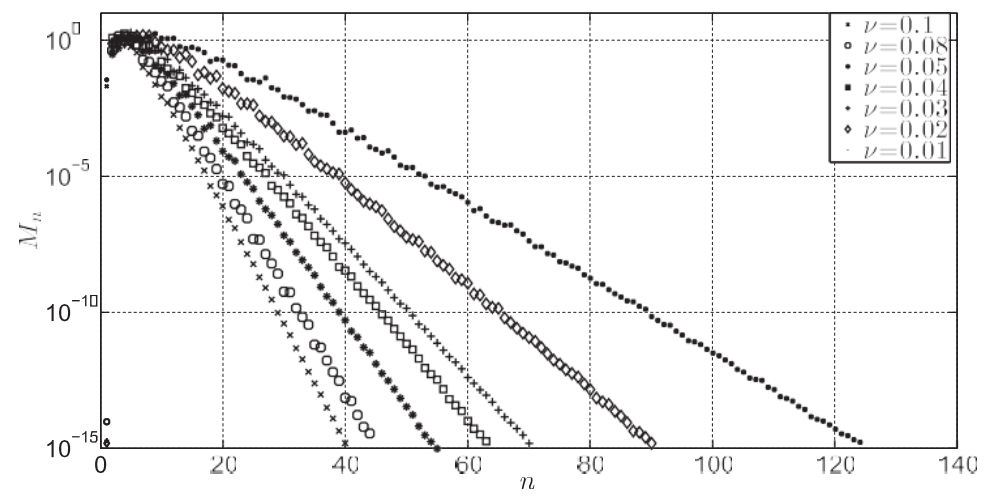

Fig. 2. $\log M_{n}$ versus $n$ showing decay of Fourier modes.

Table 1 Decay rates and band of analyticity

\begin{tabular}{lccccccc}
\hline$v$ & 0.1 & 0.08 & 0.05 & 0.04 & 0.03 & 0.02 & 0.01 \\
\hline$\beta_{V}$ & 0.986 & 0.888 & 0.729 & 0.617 & 0.553 & 0.433 & 0.314 \\
$\beta_{V} v^{-1 / 2}$ & 3.118 & 3.140 & 3.260 & 3.085 & 3.192 & 3.062 & 3.140 \\
\hline
\end{tabular}

\subsection{Decay of Fourier coefficients and analyticity estimates}

Extensive computations were carried out as $v_{1}>0$ and $v_{2}>0$ decrease, in order to establish numerically the exponential decay of the Fourier coefficients, and hence surmise analyticity of solutions. In the computations that follow, we take $\delta_{1}=\delta_{2}=0$ and $v_{1}=v_{2}=v$. Note that allowing $v_{1}, v_{2}$ to decrease at the same rate, provides the same number of unstable modes in each direction, and hence enables the development of equally complex dynamics in $x$ and $y$. At small values of $v$, the solution typically exhibits complex chaotic dynamics, and we monitor the size of the $n$th order Fourier coefficients (after sufficiently large times so that the solution enters an attractor) defined by

$$
M_{n}=\max _{|j|+|\zeta|=n}\left|\hat{u}_{j} \zeta\right|
$$

The decay of $M_{n}$ with $n$ is shown in log-linear coordinates in Fig. 2 for $0.01^{\mathrm{TM}} v^{\mathrm{TM}} 0.1$, and the results indicate that the decay is exponential; for sufficiently large $n$, we observe

$$
M_{n}=O\left(e^{-\beta_{v} n}\right)
$$

with $\beta_{v}$ increasing as $v$ decreases. The dependence of the decay rate $\beta_{v}$ on $v$ is analysed in Table 1 using least squares fits of the data of Fig. 2. The table shows the estimates of $\beta_{V}$ and $\beta_{v} v^{-1 / 2}$ as $v$ varies, and there is strong evidence to support the scaling $\beta \approx 3 v^{1 / 2}$. This in turn implies that the solution is analytic in a band of width of order $v^{1 / 2}$ as $v \rightarrow 0$; interestingly, this computational estimate is identical to that found (also numerically) in the one-dimensional Kuramoto-Sivashinsky equation-Akrivis et al. (2011, 2012), Collet et al. (1993). Note that the available analytic estimates for $\beta_{v}$ in the one-dimensional case (Collet et al., 1993; Akrivis et al., 2013), underestimate the apparently optimal $v^{1 / 2}$ value determined numerically. The present results of the two-dimensional KS equation provide an optimal bound for $\beta_{V}$ which is guiding analytical studies under way in our group. 
Table 2 Error "U(',nk) - u(',nk)" ${ }_{L^{2}}$ for $v=0.8$ at $T=1$

\begin{tabular}{|c|c|c|c|c|c|c|}
\hline$k_{1}$ & $q=1$ & $q=2$ & $q=3$ & $q=4$ & $q=5$ & $q=6$ \\
\hline 10 & $3.51 \times 10_{-1}$ & $3.48 \times 10_{-3}$ & $3.24 \times 10_{-4}$ & $3.63 \times 10_{-5}$ & $2.71 \times 10_{-6}$ & $3.13 \times 10_{-8}$ \\
\hline$\underline{20}$ & $1.88 \times 10_{-2}$ & $7.80 \times 10_{-3}$ & $4.60 \times 10_{-5}$ & $2.05 \times 10_{-6}$ & $1.10 \times 10_{-8}$ & $4.87 \times 10_{-10}$ \\
\hline 40 & $9.73 \times 10_{-2}$ & $1.83 \times 10_{-4}$ & $6.05 \times 10_{-6}$ & $1.17 \times 10_{-8}$ & $3.72 \times 10_{-9}$ & $7.63 \times 10_{-11}$ \\
\hline $8 \varphi$ & $4.94 \times 10_{-2}$ & $4.43 \times 10_{-4}$ & $7.74 \times 10_{-7}$ & $6.97 \times 10_{-9}$ & $1.20 \times 10_{-11}$ & $1.10 \times 10_{-13}$ \\
\hline 190 & $2.49 \times 10_{-2}$ & $1.09 \times 10_{-5}$ & $9.78 \times 10_{-7}$ & $4.24 \times 10_{-10}$ & $3.82 \times 10_{-12}$ & $2.70 \times 10_{-13}$ \\
\hline & $1.25 \times 10$ & $2.69 \times 10$ & $1.23 \times 10$ & $2.61 \times 10$ & $1.19 \times 10$ & $9.60 \times 10$ \\
\hline
\end{tabular}

\subsection{Accuracy tests for the six BDF schemes}

In what follows, we verify the accuracy of the schemes (2.14) for $q=1, \ldots, 6$ and in the absence of dispersion $\left(\delta_{2}=0\right)$. To achieve this, we solve the inhomogeneous equation

$$
u_{t}+u u_{x}+u_{x x}+v_{1} u_{x x x x}+2 v_{2} u_{x x y y}+\frac{\underline{v}^{2}}{v_{1}} u_{y y y y}=g(x, y, t),
$$

where the function $g(x, y, t)$ is selected so that

$$
u(x, y, t)=\cos (x+y)+\cos (t) \cos (2 x-y)
$$

solves (3.4) exactly. In all computations presented, we used $v_{1}=v_{2}=v=0.8$ and calculated the $L^{2}$ norm of the error $\left(E=" U^{n}-u^{n}{ }_{L}\right)_{2}$ between the exact and numerical solutions at time $T=1-$ the quadrature is performed with spectral accuracy in order to isolate time-stepping errors. In addition, to maintain accuracy any required starting values were found using the exact solution. The largest time step used is $k=\frac{r}{10}$ and this is halved five times to provide the smallest time step $k=\frac{1}{320}$. The results are summarized in Table 2 and the theoretical accuracy of the schemes is seen to be fully supported by the computations - the error decreases by a factor $2^{q}$ every time the time step is halved. We note that for $q=6$ the error reaches machine accuracy (double precision computations were used) when $k=160$, and hence the geometric decrease is halted and the error remains at machine precision levels. The dependence of the error on the time step $k$ for each scheme $q=1, \ldots, 6$, was also considered and plotted on logarithmic scales; the slopes of the lines increase from 1 for the first-order schemes $q=1$, to 6 for the sixth-order schemes $q=6$ (results not shown for brevity).

A second test was undertaken for the unforced equation (3.4) $(g=0)$ with $v_{1}=v_{2}=v=0.5$. In particular, we choose to numerically evaluate the theoretical accuracy characteristics of our BDF schemes on a quantitative feature of the solution such as the $L^{2}$-norm. The large time solution at this value of $v$ is a non-uniform steady state with constant energy. The convergence of the six BDF schemes to this solution as the time-step $k$ decreases, is shown in Table 3. Results are obtained by integrating the equation from random initial conditions (these are fixed for the different runs) up to a final time $T=50$ - this is chosen empirically to ensure that the solution has entered the attractor. It can be seen that for the schemes of order $q=3,4,5$ and 6 (note that these schemes are not unconditionally stable), as soon as a time step is small enough for the scheme to be stable (e.g., for $q=5$ this value is $k \approx 0.0128$ ), convergence is achieved within machine accuracy. As expected, scheme $q=1$ does not perform as well as 
Table 3 Energy for $v=0.5$ at $T=50$ (random initial conditions with coefficients fixed in all runs)

\begin{tabular}{lcccccc}
\hline$k$ & $q=1$ & $q=2$ & $q=3$ & $q=4$ & $q=5$ & $q=6$ \\
\hline 0.0512 & 13.603226 & 13.602399 & 13.602466 & 13.602466 & - & - \\
0.0256 & 13.602807 & 13.602449 & 13.602466 & 13.602466 & - & - \\
0.0128 & 13.602626 & 13.602462 & 13.602466 & 13.602466 & 13.602466 & - \\
0.0064 & 13.602544 & 13.602465 & 13.602466 & 13.602466 & 13.602466 & - \\
0.0032 & 13.602504 & 13.602466 & 13.602466 & 13.602466 & 13.602466 & 13.602466 \\
0.0016 & 13.602485 & 13.602466 & 13.602466 & 13.602466 & 13.602466 & 13.602466 \\
0.0008 & 13.602475 & 13.602466 & 13.602466 & 13.602466 & 13.602466 & 13.602466 \\
0.0004 & 13.602471 & 13.602466 & 13.602466 & 13.602466 & 13.602466 & 13.602466 \\
0.0002 & 13.602468 & 13.602466 & 13.602466 & 13.602466 & 13.602466 & 13.602466 \\
0.0001 & 13.602467 & 13.602466 & 13.602466 & 13.602466 & 13.602466 & 13.602466 \\
0.00005 & 13.602467 & 13.602466 & 13.602466 & 13.602466 & 13.602466 & 13.602466 \\
\hline
\end{tabular}

higher order schemes - indeed convergence is not seen for the smallest time-step $k=5 \times 10^{-5}$, whereas convergence is achieved for the $q=2$ scheme for time-steps smaller than $k \approx 0.0001$. The reason for this follows from the respective errors of these schemes being of order $k$ and $k^{2}$, respectively.

\subsection{Computational estimate of the size of the absorbing ball}

In Sections 3.2-3.3, we presented numerical tests which illustrate the analyticity of solutions and the accuracy and convergence of our numerical schemes. In what follows, we carry out extensive numerical experiments to obtain quantitative aspects of solutions and their absorbing set.

The first set of results is aimed at identifying numerically $L^{2}$-bounds of the size of the absorbing ball, and evaluating theoretical bounds such as those of Pinto (1999, 2001) - see (1.5). Rigorous bounds typically overestimate the radius of the absorbing ball. It is customary to present such results as the size of the domain $L_{1} \times L_{2}$ increases; we will consider $L_{1}=L_{2}=2 L$ and allow $L$ to increase - this is equivalent to $v_{1}=v_{2}=v$ decreasing in (2.1). Thus, we present numerical solutions of

$$
\begin{aligned}
& U_{t}+U U_{x}+U_{x x}+\Delta^{2} U=0, \\
& U(x+2 L, y, t)=U(x, y, t), \quad U(x, y+2 L, t)=U(x, y, t),
\end{aligned}
$$

subject to initial conditions $U(x, y, 0)=U_{0}(x, y)$. Equation (3.5) is the unscaled version of (2.1) with $\delta_{1}=\delta_{2}=0$. As $L$ increases, the number of unstable linear modes scales with $L^{2}$. This follows from the area of the unstable regions in Fig. 1 which was shown to be of order $1 / v$; the result follows by noting that $v=(\pi / L)^{2}$.

To obtain a computational estimate of the size of the absorbing ball in $L^{2}$ as the domain size $L$ increases, the initial condition $U_{0}(x, y)$ is taken to consist of a large range of linearly unstable Fourier modes with random amplitudes. Typically, the attractor becomes chaotic for $L$ sufficiently large and we calculate the time average of the energy $\left(L^{2}\right.$-norm $)$ of the solution as follows:

$$
\bar{E}(L):=\frac{1 T_{1}}{T_{2}-T_{1}}=T_{2} E(L, t) \mathrm{d} t, \quad \text { where } E(L, t)={ }_{0}{ }_{0}^{2 L>2 L} U^{2}(x, y, t) \mathrm{d} x \mathrm{~d} y .
$$




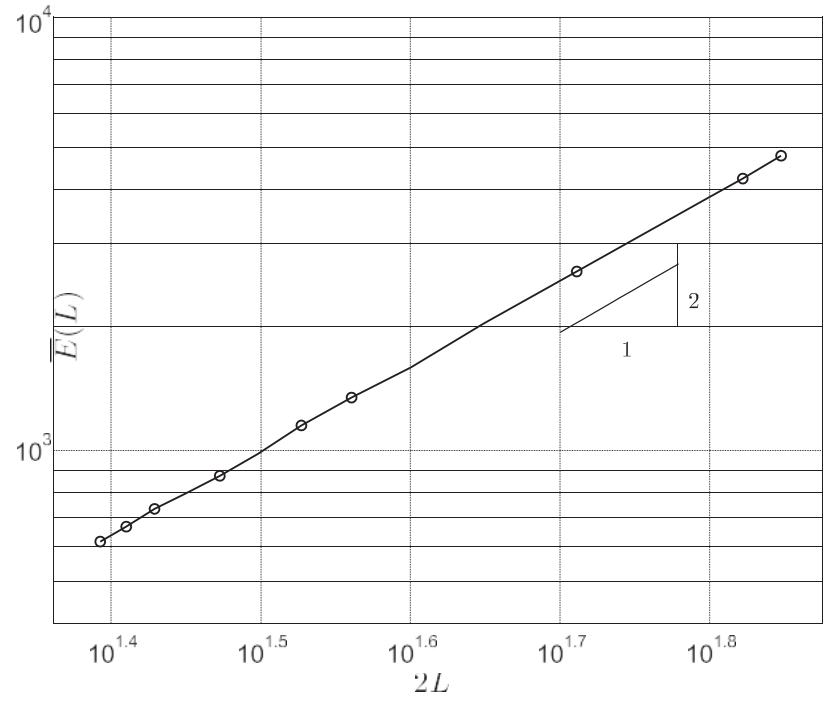

Fig. 3. $\log -\log$ plot of the time average of $L^{2}$-norms against $L$. The slope is estimated to be equal to 2 .

The times $T_{1}, T_{2}$ are chosen so as the former is sufficiently large for the solution to be inside the attractor, and the latter sufficiently large to obtain convergence to a time-average value. The results of $\bar{E}(L)$ versus $L$ are plotted using logarithmic scales in Fig. 3. The slope of $\bar{E}(L)$ provides an estimate for the energy norm of the solution as a function of $L$. Our results show conclusively that this relationship is of the form

$$
\bar{E}(L)=c L^{2}, \quad L \quad 1 .
$$

This estimate has been obtained using a least squares fit of the data presented in Fig. 3 to obtain a value of 1.9533 . To visualize the fit, we also superimpose a line of slope 2 (i.e., a function proportional to $L^{2}$ ).

Note that if higher Sobolev norms, e.g., $H^{s}, s=1,2,3$, are used in (3.6) instead of the $L^{2}$-norm, the same large $L$ behaviour shown in (3.7) is found, and in particular the slope 2 shown in Fig. 3 persists. These numerical results provide a strong indication that the two-dimensional KS equation studied here exhibits extensive dynamics analogous to the one-dimensional case (for the latter see Giacomelli \& Otto, 2005; Wittenberg \& Holmes, 1999, for example).

\subsection{Chaotic dynamics and the effect of dispersion}

In the previous sections, we carried out numerical convergence and accuracy studies of our schemes, and also established numerically a lower bound of the band of analyticity of solutions as the viscosity parameter $v$ (equivalently $v_{1}$ and $v_{2}$ ) decreases (see Section 3.2). The latter result furnishes us with a rule-of-thumb estimate for the number of modes retained in a computation as $v$ decreases-more precisely, this provides an estimate for the number of modes used in the two-thirds de-aliasing scheme. In what follows we utilize our own routines and algorithms to probe the nonlinear dynamics of the system at small values of $v$, along with the effect of the dispersion parameter $\delta_{2}$. 
(a)



(c)

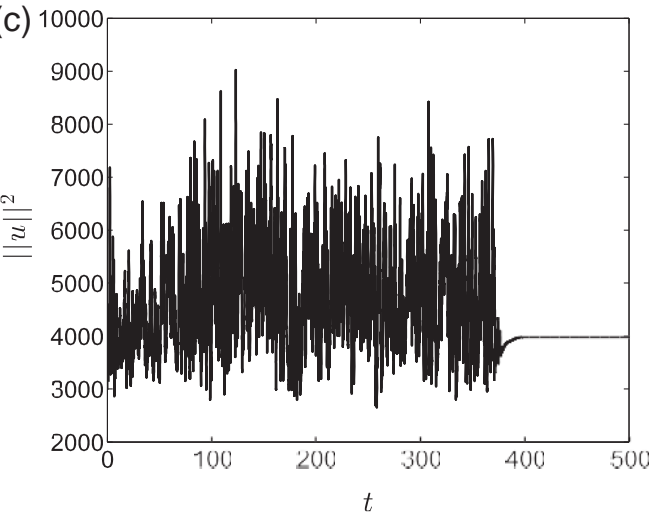

(b)

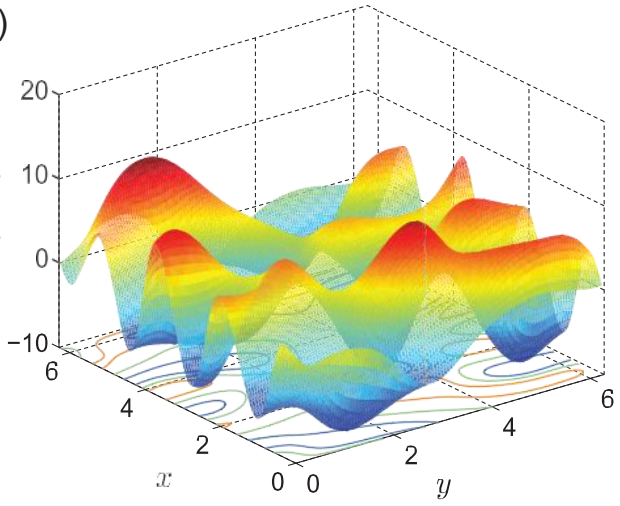

(d)



Fig. 4. $L^{2}$-norm and profile $u(x, y)$ at $t=500$ for $v=0.05$. (a and b) Chaotic solution for $\delta_{2}=0$. (c and d) Travelling wave solution for $\delta_{2}=5$. (Panels (b) and (d) color online, red high wave amplitudes, blue low wave amplitudes.)

A general finding of our computations is that the dynamics become increasingly complex, and in fact fully two dimensional and chaotic. In addition, a sufficient amount of dispersion is found to regularize chaotic dynamics into either nonlinear stable steady-state travelling wave pulses (travelling in the $x$-direction) or time-periodic ones (i.e., the dimension of the attractor is drastically reduced). Note that such regularizing dispersive effects have also been found in the one-dimensional case (see Akrivis et al., 2012, for example), and the associated reduction in dimension of the attractor as dispersion increases has not been explained analytically.

Figure 4 presents typical results for $v=0.05$ in the absence of dispersion $\delta_{2}=0$, along with the dynamics for the same value of $v$ but with $\delta_{2}=5$. Figure 4(a) shows the evolution of the energy norm over a time interval $0^{\mathrm{TM}} t^{\mathrm{TM}} 500$, and in Figure 4(b) we present the corresponding solution at the final time $t=500$. These results strongly suggest the presence of spatiotemporal chaos; numerical confirmation has been obtained by constructing return maps of the energy extrema, as described in detail for the one-dimensional KS equation in Akrivis et al. (2011). Figure 4(c,d) depicts analogous results corresponding to $v=0.05$ but $\delta_{2}=5$. We observe that the dynamics initially enter a chaotic attractor for a time interval of $\sim 400$ time units, but beyond this time the effects of dispersion are felt, and transform the solution into a nonlinear travelling wave composed of two pulses in the $x$-direction and three pulses 
in the $y$-direction. We emphasize that the computed solution is stable at these values of $\delta_{2}$, but eventually loses stability at higher $\delta_{2}$. In a related study, Saprykin et al. (2005) construct travelling wave solutions by assuming solutions of permanent form, i.e., $u(x, y, t)=u(x-c t, y)$, but do not provide information about their stability. Our computations (based on initial value problems) suggest that most of these travelling wave solutions are unstable in the sense that they do not emerge as large time solutions to the initial value problem. This facet of the two-dimensional problem is quite distinct from the behaviour in the one-dimensional case, where even moderate amounts of dispersion produce travelling wave pulses, see Akrivis et al. (2012).

\section{Conclusions}

In this study we introduced, analysed and implemented a class of space-time schemes to solve twodimensional PDEs arising in viscous falling film flows. The equations (known as the Topper-Kawahara equations) contain nonlinear advection in the stream wise $x$-direction, negative diffusion terms due to inertial effects (we only consider situations above critical, $F^{2}>\frac{5}{2}$, where $F$ is the Froude number), twodimensional dispersive effects and two-dimensional dissipation due to the presence of surface tension.

Global existence of solutions, the existence of a global attractor, and analyticity has been proven by Pinto (1999, 2001), who also provides a bound for the radius of the absorbing ball in the $L^{2}$-norm. Such analytical bounds are far from optimal, and we have carried out accurate computations to numerically determine the absorbing ball radius as the system size $L$ increases and the dynamics enter a strongly chaotic regime. Our results show that the radius of the global attractor has size $L$ (see (1.6) and (3.7)), i.e., scales with the linear scale of the physical computational domain. Our computations also show that the solutions remain analytic in a band of width $1 / L$ as $L$ increases, and again this provides an optimal bound that can guide analytical studies.

The overall features of the dynamics have been obtained, and it is established that two-dimensional spatio-temporal chaos persists as the system size $L$ increases. It has also been illustrated that when dispersion is present in the deeply chaotic regime, it acts to regularize the dynamics in the sense that it has a propensity to reduce the dimension of the global attractor, and to produce two-dimensional structures such as time-periodic travelling waves or nonlinear travelling waves of permanent form consisting of arrays of pulses - see Fig. 4(d) for an illustrative example.

\section{Acknowledgements}

This work was partly supported by an internal University of Cyprus grant. The work of DTP was partly supported by the EPSRC grant number EP/K041134/1.

\section{References}

Adams, R. A.\& Fournier, J. J. F. (2003) Sobolev Spaces, 2nd edn. Amsterdam: Academic Press.

Akrivis, G. \& Crouzeix, M. (2004) Linearly implicit methods for nonlinear parabolic equations. Math. Comp., 73, 613-635.

Akrivis, G., Crouzeix, M. \& Makridakis, Ch. (1999) Implicit-explicit multistep methods for quasilinear parabolic equations. Numer. Math., 82, 521-541.

Akrivis, G., Papageorgiou, D. T. \& Smyrlis, Y.-S. (2011) Linearly implicit methods for a semilinear parabolic system arising in two-phase flows. IMA J. Numer. Anal., 31, 299-321.

Akrivis, G., Papageorgiou, D. T. \& Smyrlis, Y.-S. (2012) Computational study of the dispersively modified Kuramoto-Sivashinsky equation. SIAM J. Sci. Comp., 34, A792-A813. 
Akrivis, G., Papageorgiou, D. T. \& Smyrlis, Y.-S. (2013) On the analyticity of certain dissipative-dispersive systems. Bull. Lond. Math. Soc., 45, 52-60.

Akrivis, G. \& Smyrlis, Y.-S. (2011) Linearly implicit schemes for a class of dispersive-dissipative systems. Calcolo, 48, 145-172.

Boyd, J. P. (2001) Chebyshev and Fourier Spectral Methods, 2nd edn, revised. Mineola, NY: Dover Publications.

Canuto, C., Hussaini, M. Y., Quarteroni, A. \& Zang, T. A. (1988) Spectral Methods in Fluid Dynamics. Springer Series in Computational Physics. New York: Springer.

Collet, P., Eckmann, J.-P., Epstein, H.\& Stubbe, J. (1993) Analyticity for the Kuramoto-Sivashinsky equation. Physica D, 67, 321-326.

Frenkel, A. L. \& Indireshkumar, K. (1999) Wavy film flows down an inclined plane: perturbation theory and general evolution equation for the film thickness. Phys. Rev. E (3), 60, 4143-4157.

Frost, F. \& Rauschenbach, B. (2003) Nanostructuring of solid surfaces by ion-beam erosion. Appl. Phys. A, 77, 1-9.

Giacomelli, L. \& Otto, F. (2005) New bounds for the Kuramoto-Sivashinsky equation. Commun. Pure Appl. Math., 58, 297-318.

Indireshkumar, K. \& Frenkel, A. L. (1997) Mutually penetrating motion of self-organized two-dimensional patterns of soliton-like structures. Phys. Rev. E, 55, 1174-1177.

Kassam, A.-K. \& Trefethen, L. N. (2005) Fourth order time-stepping for stiff PDEs. SIAM J. Sci. Comput., 26, 1214-1233.

Molinet, L. (2000) Local dissipativity in $L^{2}$ for the Kuramoto-Sivashinsky equation in spatial dimension 2. J. Dyn. Diff. Equat., 12, 533-556.

Pinto, F. C. (1999) Nonlinear stability and dynamical properties for a Kuramoto-Sivashinsky equation in space dimension two. Discr. Cont. Dyn. Syst., 5, 117-136.

Pinto, F. C. (2001) Analyticity and Gevrey class regularity for a class of Kuramoto-Sivashinsky equation in space dimension two. Appl. Math. Lett., 14, 253-260.

Saprykin, S., Demekhin, E. A. \& Kalliadasis, S. (2005) Two-dimensional wave dynamics in thin films. I. Stationary solitary pulses. Phys. Fluids, 17, 1-16.

Sell, G. R. \& Taboada, M. (1992) Local dissipativity and attractors for the Kuramoto-Sivashinsky equation in thin 2D domains. Nonlinear Anal., 18, 671-687.

Tadmor, E. (1986) The well-posedness of the Kuramoto-Sivashinsky equation. SIAM J. Math. Anal., 17, 884-893.

Topper, J. \& Kawahara, T. (1978) Approximate equations for long nonlinear waves on a viscous fluid. J. Phys. Soc. Jpn, 44, 663-666.

Wittenberg, R. W. \& Holmes, P. (1999) Scale and space localization in the Kuramoto-Sivashinsky equation. Chaos, 9, 452-465. 